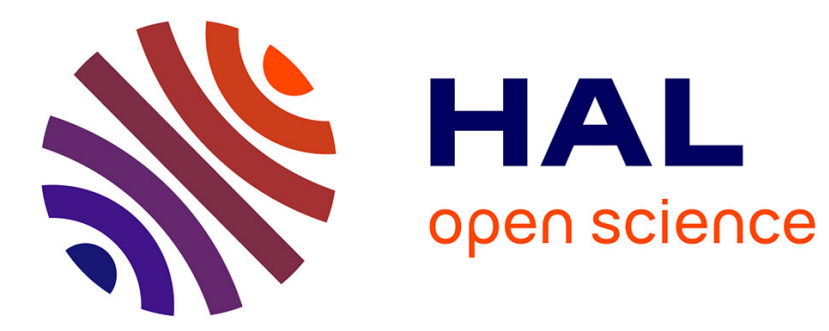

\title{
In vivo imaging of tagged mRNA in plant tissues using the bacterial transcriptional antiterminator BglG
}

\author{
Eduardo Pena, Gabriel Robles Luna, Manfred Heinlein
}

\section{To cite this version:}

Eduardo Pena, Gabriel Robles Luna, Manfred Heinlein. In vivo imaging of tagged mRNA in plant tissues using the bacterial transcriptional antiterminator BglG. Plant Journal, 2020, 10.1111/tpj.15035 . hal-03022805

\section{HAL Id: hal-03022805 \\ https://hal.science/hal-03022805}

Submitted on 24 Nov 2020

HAL is a multi-disciplinary open access archive for the deposit and dissemination of scientific research documents, whether they are published or not. The documents may come from teaching and research institutions in France or abroad, or from public or private research centers.
L'archive ouverte pluridisciplinaire HAL, est destinée au dépôt et à la diffusion de documents scientifiques de niveau recherche, publiés ou non, émanant des établissements d'enseignement et de recherche français ou étrangers, des laboratoires publics ou privés. 


\section{The Plant Journal - TECHNICAL ADVANCE}

\section{Title}

In vivo imaging of tagged mRNA in plant tissues using the bacterial transcriptional antiterminator BglG

\section{Authors}

Eduardo José Peña ${ }^{1,2, *}$, Gabriel Robles² Luna and Manfred Heinlein ${ }^{1 *}$

${ }^{1}$ Université de Strasbourg, CNRS, IBMP UPR 2357, F-67000 Strasbourg, France

2Instituto de Biotecnología y Biología Molecular (IBBM), Facultad de Ciencias Exactas, CONICET UNLP, La Plata, Argentina

${ }^{*}$ Address correspondence to:

Eduardo Peña (eduardop@biol.unlp.edu.ar)

Manfred Heinlein (manfred.heinlein@ibmp-cnrs.unistra.fr)

Running Title: RNA imaging with BglG

\section{Keywords}

RNA localization, RNA transport, BglG, MS2, $\lambda \mathrm{N}, \mathrm{TMV}$, movement protein, cell-to-cell movement, RNA labeling, RNA imaging. 


\section{Summary}

RNA transport and localization represent important post-transcriptional mechanisms to determine the subcellular localization of protein synthesis. Plants have the capacity to transport mRNA molecules beyond the cell boundaries through plasmodesmata (PD) and over long distances in the phloem. RNA viruses exploit these transport pathways to disseminate their infections and represent important model systems to investigate RNA transport in plants. Here, we present an in vivo plant RNA-labeling system based on the E.coli RNA-binding protein BglG. Using the detection of RNA in mobile RNA particles formed by viral movement protein (MP) as a model, we demonstrate the efficiency and specificity of mRNA detection by the BgIG system as compared to MS2 and $\lambda \mathrm{N}$ systems. Our observations show that MP mRNA is specifically associated with MP in mobile MP particles but hardly with MP localized at PD. MP mRNA is clearly absent from MP accumulating along microtubules. We show that the in vivo BgIG labeling of the MP particles depends on the presence of the BglG-binding stem-loop aptamers within the MP mRNA and that the aptamers also enhance the coprecipitation of BgIG by MP, thus demonstrating the presence of a MP:MP mRNA complex. The BgIG system also allowed us to monitor the cell-to-cell transport of the MP mRNA, thus linking the observation of mobile MP mRNA granules with intercellular MP mRNA transport. Given its specificity demonstrated here, the BgIG system may be widely applicable for studying mRNA transport and localization in plants. 


\section{Introduction}

mRNA localization is an evolutionary conserved mechanism observed in a wide range of organisms and cell types, and by which mRNA species carrying RNA localization signals ("zipcodes") are localized to discrete locations in the cytoplasm (Ryder and Lerit, 2018). By dictating the specific localization at which encoded proteins are translated and operate, RNA localization processes play central roles in the determination of cell polarity, embryonic patterning, asymmetric cell-fate decisions, neuronal morphogenesis, cell migration, or epithelial polarity (Ryder and Lerit, 2018). Initial evidence for RNA compartmentalization was based on laborious in situ hybridization methods for the detection of individual mRNAs (Jeffery et al., 1983). In more recent years the advent of transcriptome-wide approaches showed that RNA localization is much more widespread than previously assumed, thus indicating that RNA localization has a prominent role in the spatial regulation of gene activity (Lécuyer et al., 2007; Holt and Bullock, 2009). RNA localization is a multistep process involving mRNA export from the nucleus, protection from degradation, cytoplasmic transport, and localized anchorage. Upon transcription and mRNA maturation, the zipcodes are recognized by RNA-binding proteins and play important roles during nuclear export and cytoplasmic sorting (Eliscovich and Singer, 2017). It is accepted that localized RNAs are transported as ribonucleoprotein complexes (RNPs) or "RNA granules". These granules are transported by molecular motors along the cytoskeleton to their final destination, where the mRNAs are anchored and eventually translated (Holt and Bullock, 2009; Martin and Ephrussi, 2009; Buxbaum et al., 2015; Mofatteh and Bullock, 2017).

Cell-to-cell communication is a fundamental process essential for the distribution of signaling molecules during the coordination of developmental programs and during adaptation to environmental or intrinsic stimuli. Plants allow intercellular transport and exchange of nutrients and signaling molecules through plasmodesmata (PD). These gatable 
membranous pores extend through the cell wall and provide cytoplasmic and membrane continuity between cells (Zambryski and Crawford, 2000; Heinlein, 2002). The system of PD is connected to the phloem sieve elements, thus forming a cell-to-cell and long-distance communication network (Lucas and Lee, 2004). Similar to other systems, also plants have the capacity to transport and localize RNA molecules to specific cellular compartments (Okita and Choi, 2002; Tian et al., 2020). Moreover, given the communication network established by PD and connected phloem, RNA molecules can also move between cells and tissues (Kehr and Buhtz, 2008; Nazim Uddin and Kim, 2013; Kehr and Kragler, 2018). Although PD are essential for intercellular communication and plant life, the underlying mechanisms by which RNA molecules are targeted to PD and transported between cells remain poorly understood. One way to address these mechanisms is by studying the cellto-cell transport of single-stranded RNA viruses. Viruses encode specialized proteins known as movement proteins (MPs) that localize to PD, modify PD regulation and allow the passage of the viral RNA (Heinlein, 2015; Peña and Heinlein, 2012). Tobacco mosaic virus (TMV), undoubtedly the most studied plant virus (Scholthof et al., 2011), is a singlestranded, positive-sense RNA virus. The viral genome encodes two subunits of the replicase as well as the MP and the coat protein (CP) (Goelet et al., 1982). TMV cell-to-cell movement is independent of CP (Holt and Beachy, 1991) and likely occurs as a non-encapsidated ribonucleoprotein complex (RNP) that contains viral RNA in association with MP and replicase (Heinlein, 2015). Therefore, studies on TMV movement promise to reveal the mechanisms underlying the intra- and intercellular transport of RNA complexes in plants (Peña and Heinlein, 2012).

The use of TMV derivatives that express MP as a fusion to fluorescent reporters led to the discovery that the protein interacts with the endoplasmic reticulum (ER) and the microtubule (MT) cytoskeleton (Heinlein et al., 1995; Heinlein et al., 1998). Several subsequent studies showed that the interaction with MTs is tightly associated with MP function in virus 
movement (Boyko et al., 2000a; Boyko et al., 2007; Ouko et al., 2010; Niehl et al., 2013). Conditional mutations in MP were used to correlate the ability of MP to support the cell-tocell movement of the virus with the formation of MP particles showing stop-and-go movements in the cortical cytoplasm (Boyko et al., 2000b; Boyko et al., 2007; Boyko et al., 2002). Mobile MP particles are also formed during ectopic expression of MP and their further analysis revealed that the particles localize to the cortical ER-actin network, at positions associated with intersecting MTs ("cortical MT-associated ER sites", cMERs (Peña and Heinlein, 2013)). The movements of the particles occur along the ER-actin network, apparently guided by MTs and pausing their trajectories at ER-MT intersection points (Sambade et al., 2008). Further studies demonstrated that TMV movement and the targeting of MP to PD depends on specific class XI and class XIII myosins (Amari et al., 2014). The association of MP particles with cMERs is consistent with the association of MP with membranes (Peiro et al., 2014) and its affinity to bind MTs (Ashby et al., 2006) and MTassociated proteins (Brandner et al., 2008; Ferralli et al., 2006). Further studies suggested that the cMERs and PD may share features of ER:PM contact sites and that these features may be targeted for localization of MP and the MP-associated viral RNA (Pitzalis and Heinlein, 2017). A likely candidate for such a common feature is synaptotagmin 1 (SYT1). This protein occurs at ER:PM contact sites as well as at PD (Ishikawa et al., 2020; Levy et al., 2015) and is a target of MP required for virus movement (Levy et al., 2015; Yuan et al., 2018; Lewis and Lazarowitz, 2010)

While proteins can be localized by translational fusion to fluorescent reporters, the development of comparable techniques to localize RNA molecules by transcriptional fusion to a fluorescent RNA molecule remain to be developed. Instead, RNA molecules are observed upon in vitro labeling with fluorochromes and delivery to cells by transfection or microinjection (Wilkie and Davis, 2001), or upon in vivo labeling by indirect methods. These indirect methods are based either on hybridization of native RNA molecules with fluorescent 
probes that are delivered into the cell, or on the use of RNA-binding proteins (RBPs) or chemical ligands that tag the RNA upon binding to RNA motifs transcriptionally fused to the RNA under study. Whereas the RBPs are used as fusions to fluorescent proteins to image the localization of the tagged RNA, the chemical ligands become by themselves fluorescent upon binding to the RNA (Tilsner, 2015; George et al., 2018; Christensen et al., 2010). One of the most frequently applied RNA visualization techniques in animal cells is the MS2 method (Bertrand et al., 1998). This method is based on the high affinity binding of the MS2 coat protein (MS2-CP) to a specific RNA stem-loop (MS2-SL) structure that initiates encapsidation of the bacteriophage RNA genome. The MS2 method utilizes a tandem array of this SL by transcriptionally fusing it to the mRNA under investigation and by expressing the SL-tagged mRNA together with the MS2-CP translationally fused to a fluorescent reporter, such as GFP. In order to reduce cytoplasmic background, the MS2-CP:GFP protein is fused to a nuclear localizing sequence (NLS). Thus, once transcribed, the mRNA carrying the SL array will be recognized and bound by the RBP (NLS:MS2-CP:GFP) forming a complex exported to the cytoplasm as a trackable fluorescent mRNP (Bertrand et al., 1998). Using the MS2 system in plants, an SL array-tagged mRNA encoding the MP of TMV in fusion to red fluorescent protein (RFP) was shown to localize to the mobile MP particles in the cortical cytoplasm (Sambade et al., 2008). The mRNA signal in the MP particles depended on the presence of the SL array in the MP mRNA, thus showing that the signal reported by NLS:MS2-CP:GFP was specific (Sambade et al., 2008). Moreover, the tagged MP mRNA was also observed to colocalize with MP at the PD. These findings indicated that the MP particles contain MP mRNA and that the MP facilitates the targeting of its own mRNA to the PD (Sambade et al., 2008). However, although these results were robust, the sensitivity of the method was low. Thus, only a small number of RNA-containing MP particles could be detected. 
To improve the sensitivity of RNA detection, we tested two other RNA labeling systems. Whereas the ' $\lambda \mathrm{N}$ system' uses the antitermination protein $\mathrm{N}$ of bacteriophage lambda as RBP (Daigle and Ellenberg, 2007), another system that was not previously used in plants uses the antiterminator protein $\mathrm{G}$ of the $E$. coli $\mathrm{bgl}$ operon as RBP and is referred to as the 'BglG system' (Chen et al., 2009). Both systems allowed us to confirm the presence of MP mRNA in MP particles and to detect MP mRNA together with MP at PD. With the aim to further increase the strength of the specific mRNA signal, we decided to increase the cytoplasmic accumulation of the RBPs by removing the NLS. Whereas the MS2 and $\lambda N$ RBPs formed large cytoplasmic aggregates under these conditions, the RBP of the BgIG system showed a homogenous cytoplasmic distribution. The BglG protein was still homogenously distributed in the cytoplasm when co-expressed with MP. However, this localization changed dramatically when the MP encoding mRNA carried the cognate BgIGbinding SLs. In this case, BgIG was recruited to multiple mobile MP particles but not to highly MP-decorated MTs, thus specifically identifying the MP particles as RNPs. Coimmunoprecipitation experiments confirmed these observations by showing that BgIG coprecipitates efficiently with MP if MP is expressed from a SL-tagged mRNA but not if MP is expressed from mRNA without the SLs. Furthermore, we show that the BglG-labeled mRNA moves cell-to-cell thus linking the detection of cytoplasmic, mRNA-containing, mobile MP particles with mRNA movement.

\section{Results}

\section{Imaging of MP mRNA with MS2, $\lambda N$ and BgIG systems}

Using the MS2 method to study TMV RNA transport and localization, it was shown that MP colocalizes with MP mRNA (tagged with 12 MS2-SLs) in mobile particles and at PD, thus 
suggesting that the mobile particles are RNPs destined to PD (Sambade et al., 2008). However, further studies were hampered by the low sensitivity of mRNA detection. When we repeated these experiments, the localization of the MP mRNA at PD was observed regularly, whereas the association of the mRNA with the mobile MP particles in the cytoplasm was much harder to detect (Figure 1a and Movie S1). Increasing MP expression to levels observed during infection compromised the background of our system. Therefore, to achieve RNA visualization with higher sensitivity and resolution, we decided to test other RNA localization methods.

We started by testing the $\lambda \mathrm{N}$ system, which is based on the specific binding of a 22 amino acids long peptide derived from the phage lambda protein $N(\lambda N, 3.1 \mathrm{kDa})$ to an RNA hairpin formed by 15 nucleotides (boxB) derived from the nut $\mathrm{N}$ binding site in the $N$ mRNA. Similar to the MS2 system, the $\lambda \mathrm{N}$ system allows mRNA detection by fusing the mRNA of interest with boxB SLs and by co-expression of the RBP $(\lambda N)$ targeted to the nucleus and fused to a fluorescent reporter (Daigle and Ellenberg, 2007; Schönberger et al., 2012). Coexpression of the RBP ( $\lambda \mathrm{N}: \mathrm{eGFP}: \mathrm{NLS})$ with MP:mRFP carrying a transcriptional fusion to 16 copies of the boxB-SLs (MP:mRFP-boxB) led to detection of the MP:mRFP mRNA in MP particles (Figure $1 \mathrm{~b}$ ), thus confirming that mobile MP particles indeed contain MP mRNA (Movie S2). The MP mRNA was also detected at PD together with MP, albeit with low frequency (Figure 1b).

As a second alternative, we tested the BglG system. This system has been developed for the detection of HIV RNA within HIV virions (Chen et al., 2009) and has not been applied to plants. We adapted this system to plants by translationally fusing 57 aminoacids of the Nterminal portion of the BglG protein (BglG, $6.3 \mathrm{kDa}$ ) carrying the RNA-binding domain to the red fluorescent protein mCherry and to an SV40 NLS. The resulting RBP construct, BgIG:mCherry:NLS, was then expressed together with MP:GFP encoded by mRNA tagged 
with 18 copies of the $29 \mathrm{nt}$ long BgIG-SL. This again led to the localization of the MP mRNA in MP particles (Figure 1c and Movie S3). However, this method barely detected any MP mRNA-specific signal at PD (Figure 1c).

The three tested RNA labeling methods allowed the imaging of MP mRNA colocalizing with MP particles in the cell cortex. Some of these MP RNA-containing MP particles remained stationary while other particles where mobile and displayed a stop-and-go movement, as previously noted (Sambade et al., 2008). In between short stationary intervals, the particles showed movements that alternated between episodes of random motion over short distances at intermediate velocities and directed movements for long trajectories with higher velocities of up to $3-3,5 \mu \mathrm{m} / \mathrm{s}$ (Figure S1). These intervals of MP particle movement at higher velocities are compatible with the reported velocities of myosin-driven transport (Nebenführ et al., 1999) and are also consistent with the role of class XI myosins in the targeting of MP to PD as well as in TMV movement (Amari et al., 2014).

\section{Improving sensitivity of mRNA detection}

We wondered whether we could achieve stronger RNA labeling by removal of the NLS sequences and thereby increasing the cytoplasmic level of the RBP. However, expression of either MS2-CP:GFP or $\lambda \mathrm{N}$ :GFP without NLS sequences and in the absence of the specific RNA binding target led to aberrant and unspecific accumulations of the proteins in the cytoplasm. Both proteins accumulated in the nucleus and in irregular cytoplasmic bodies visible already at low magnification (Figure 2a, left and center). In contrast, BglG:mRFP did not accumulate in any particularly dense cytoplasmic location and showed a clear homogenous nucleocytoplasmic distribution (Figure 2a, right). Imaging cortical planes at high magnification demonstrated that MS2-CP:GFP and $\lambda \mathrm{N}$ :GFP were seen in the form of small granules with high motility, thus structures that undoubtedly could mask the specific labeling and detection of any SL-tagged RNA (Figure 2b, left and center panels; Movies S4 
and S5). In contrast, BglG:mRFP showed a homogenous distribution in the cortical cytosol (Figure 2b, right panel), with movements according to cytoplasmic streaming (Movie S6). Thus, unlike MS2-CP:GFP and $\lambda \mathrm{N}$ :GFP, BgIG:mRFP turned out to be suitable for expression without NLS in further RNA labeling experiments.

When co-expressed with MP:GFP translated from untagged MP:GFP mRNA (without BgIG SLs), BgIG:mRFP retained its homogenous distribution in the cytoplasm (Figure 3a). The BgIG:mRFP fluorescence again followed cytoplasmic streaming (Movie S6 and Movie S7) independent of MP:GFP that typically decorated MTs or localized to particles showing intermittent movement along or between MTs, as previously described (Sambade et al., 2008; Boyko et al., 2007). In contrast, expression of MP:GFP from BglG-SL-tagged MP:GFP mRNA (MP:GFP-SL) led to recruitment of BgIG:mRFP to the MP:GFP particles (Figure 3ab; Movie S7 and Movie S8) resulting in the labeling of the RNP granules with high contrast (Figure 3b). Fluorescence signal intensity analysis confirmed this specific recruitment of BgIG:mRFP to the SL-tagged RNA (Figure S2). Interestingly, the RNA signal colocalized exclusively with MP within MP particles and was absent from MP localized along MTs. This observation confirms the role of MP particles as RNPs and also shows that the MP by itself either is insufficient for in vivo RNA binding or exists in different isoforms, an RNA bindingcompetent form present in the mobile MP particles and an RNA binding-incompetent form localized to MTs. The homogeneous, non-localized distribution of BglG:mRFP in the absence of its cognate RNA-SL is also observed in median planes of the cell, in close proximity to MP:GFP localized at PD (Figure 3c, arrowheads). In contrast, in the presence of MP:GFP tagged with of BgIG-SLs, BgIG:mRFP co-localized with MP:GFP at the PD, thus indicating an association of MP:GFP mRNA with PD-localized MP:GFP (Figure 3d, arrows). However, several of the MP:GFP spots occurred without a corresponding RNA labeling (Figure 3d, arrowheads), indicating that MP:GFP mRNA does not always colocalize with MP:GFP at the PD. We did not observe any significant difference in the motions of free- and 
BglG-tagged MP:GFP particles, suggesting that neither the RNA tag nor the binding of the RBP to this tag affects particle behavior (Figure S3).

While agroinfiltration ensures strong protein expression in almost all the cells of the infiltrated $N$. benthamiana leaf patch (Kapila et al., 1997), conditions need to be optimized for protein co-expression experiments. To facilitate the visualization of tagged RNA molecules, we developed a BgIG:mRFP-transgenic $N$. benthamiana line. Transient expression of MP:GFP or MP:GFP-SL in this line revealed the presence of MP:GFP mRNA in MP:GFP particles, and, as expected, the mRNA signal in the particles was dependent on the SLs and, thus, absent when the MP:GFP construct without SLs was used (Figure S4).

The requirement of the SLs for efficient binding of BgIG:mRFP to the MP:GFP mRNA was further confirmed by in vitro experiments showing that the presence of the SLs within the MP:GFP mRNA enhances the co-precipitation of BglG:mRFP by MP:GFP from plant extracts above experimental background (Figure $3 e$ and f).

\section{MP mRNA moves cell-to-cell}

Having shown that the BglG system can be used for mRNA detection and the analysis of mRNA movements inside cells, we next wanted to know if the same approach can also be used for the detection of mRNA movements between cells. A hallmark feature of viral MPs is their ability to modify the size exclusion limit of PD and to translocate through PD into the neighboring cells (Lucas, 2006). The cell-to-cell movement of MPs can be monitored in N. benthamiana leaves upon expression in isolated cells using diluted agrobacterium cultures for infiltration (Robles Luna et al., 2013; Brunkard et al., 2015), however, to our knowledge, such a simple in vivo method has not been reported for assessing the cell-to-cell trafficking of mRNA molecules. Instead, previous approaches used fluorescent in vitro-labeled RNA molecules that were delivered into the cell by microinjection. Such assays demonstrated that E. coli produced cucumber mosaic virus MP facilitated the cell-to-cell movement of co- 
injected, fluorescently-labelled KNOTTED mRNA (Lucas et al., 1995). To determine whether the observed MP mRNA-containing MP particles are associated with intercellular movement of MP mRNA, we performed co-agroinfitration experiments in which we combined high concentrations of agrobacterium $\left(\mathrm{OD}_{600}=0,4\right)$ for the expression of MP:GFP or MP:GFP. $S L$ in all cells of the infiltrated tissue with highly diluted cultures $\left(\mathrm{OD}_{600}=1,0 \times 10^{-4}\right)$ for coexpression of BgIG:mRFP only in isolated cells. We expected that when MP mRNA is transported from a cell that expresses BgIG:mRFP to neighboring cells that do not express this protein, BglG:mRFP should highlight the transported mRNA by co-transport of its red fluorescent signal due to its binding to the BgIG SLs in the mRNA. Figure 4a shows that BglG:mRFP is indeed transported between cells and that this event depends on the presence of BglG-SLs in the MP:GFP mRNA. We considered a cell-to-cell transport event when two or more neighboring cells showed accumulation of BglG:mRFP fluorescence. From all the red fluorescent, BglG:mRFP-containing spots, only $11 \%$ showed red fluorescence in more than one cell when the RBP was expressed together with MP:GFP from untagged MP:GFP mRNA. In contrast, this number increased to $32 \%$ when BgIG:mRFP was expressed together with MP:GFP-SL, thus in the presence of BgIG-SL tagged mRNA (Figure 4b and Table S1). Considering that the distribution of independent transformation events upon infiltration of a low concentration of agrobacteria follows the Poisson distribution, the occurrence of adjacent cells that express BglG:mRFP to a frequency of $32 \%$ of all transformation events by independent transformation of the adjacent cells is unlikely ( $P<2,3 \times 10^{-13}$; see Materials and Methods). The increased frequency of adjacent cells that contain BgIG:mRFP seen in the presence of SL-tagged MP:GFP mRNA therefore suggests the visualization of intercellular, MP-facilitated RNA transport. The occurrence of foci with more than one cell showing mRFP fluorescence $(11 \%)$ in the absence of the SL tags may indicate a background of intercellular BglG:mRFP (33 kDa) diffusion as similar values were previously observed for the cell-to-cell movement of free 
GFP (27 kDa) (Borniego et al., 2016; Robles Luna et al., 2013). In essence, the observations indicate that (a) transiently expressed MP mRNA is transported between cells, (b) this event can be imaged with the BglG system presented here, and (c) mRNA movement occurs in the form of an RNP complex into which BglG:mRFP is incorporated and co-transported through PD.

\section{Discussion}

Traditionally, mRNAs have been viewed as simple intermediates between the genomic information stored in the DNA and the encoded effector proteins. It is now clear that mRNAs are actively localized to distinct subcellular domains, with fundamental implications in embryonic patterning, asymmetric cell division, and cell polarity and, thus, in the determination of cell fate (Chin and Lécuyer, 2017; Buxbaum et al., 2015). Plant mRNAs are also able to move between cells and to distant tissues, and this trafficking plays important roles in the regulation of plant development, growth, and defense (Ham and Lucas, 2017). Grafting experiments followed by high throughput transcriptomic analysis demonstrated that more than 2000 genes produce phloem-mobile mRNAs in Arabidopsis (Thieme et al., 2015). Similar numbers of transported mRNAs where also identified following analysis of grafted grapevines or cucumber (Yang et al., 2015; Zhang et al., 2016). Phosphate stress treatments to cucurbit-grafted plants identified a population of transcripts involved specifically in early phosphate stress signaling. In addition, some of these molecules were selectively transported from the rootstock to specific tissues on the scion, thus highlighting specific signaling roles of mRNA transport (Zhang et al., 2016). However, while an increasing number of phloem-mobile RNA molecules is being identified and general features and functions are being elucidated (Liu and Chen, 2018; Kehr and Kragler, 2018; Reagan 
et al., 2018; Yang et al., 2019), the mechanisms underlying the selective entry of mRNAs into the phloem long-distance stream remains difficult to address. The transporting phloem sieve elements are devoid of nuclei. Thus, in order to function in signaling, the mRNAs must enter and exit the phloem sieve elements through PD, requiring active intracellular transport to these channels. Understanding the mechanism by which mRNAs are transported between cells and systemically requires approaches to visualize mRNAs and their transport complexes with subcellular resolution.

The MS2 method is broadly used to visualize RNA molecules in organisms ranging from bacteria to higher eukaryotes and to study the processes of transcription, transport, translation and decay (Bertrand et al., 1998; Vera et al., 2016; Tutucci et al., 2018; Grünwald and Singer, 2010; Larson et al., 2011; Wu et al., 2016). The method has also been applied in plants and allowed to localize specific mRNAs and virus-derived RNAs (Sambade et al., 2008; Luo et al., 2018; Michaud et al., 2014; Hamada et al., 2003). In this work, we compared the MS2 system with the $\lambda \mathrm{N}$ and BgIG systems for the ability to label MP mRNA within mobile MP particles and MP-labelled PD (Figure 1). While all these systems equally suffer from low sensitivity, the sensitivity of the BgIG system could be increased by expressing the BgIG protein without NLS, thus by augmenting its cytoplasmic concentration. This approach failed with the $\lambda N$ and MS2 systems because the $N$ and CP proteins formed cytoplasmic aggregates and could not be used under these conditions. Thus, using the BglG system under conditions of high levels of the BgIG protein in the cytoplasm, we could distinguish the presence of MP mRNA in mobile MP granules and its absence from MP localized to other sites, thus clearly identifying the MP granules as RNA particles. The specific role of the SLs for efficient MP mRNA detection was confirmed by coimmunoprecipitation experiments showing that SLs in the MP:GFP mRNA strongly enhance the coprecipitation of BgIG:mRFP with MP. By using affinity beads for MP:GFP, the coprecipitation of BglG:mRFP suggests 
the binding of BgIG to a MP:MP mRNA-SL complex (Figure 3e), which is consistent with the observed in vivo detection of SL-tagged MP RNA in MP granules.

Previous results obtained with the MS2 system indicated that MP accumulates with its own RNA at PD (Sambade et al., 2008). While our adapted BglG system allowed the detection of MP mRNA within MP particles with unprecedented sensitivity, the BglG:mRFP rarely localized with SL-tagged mRNA to PD. However, given that BgIG:mRFP showed cell-to-cell movement together with the bound MP mRNA, it is understandable that mRNA may not accumulate in PD but rather moves through the channel without being retained in the channel cavities. The reason why the MS2 system shows more frequent colocalization of $M P$ mRNA with MP protein in PD than the BglG system is unclear. However, unlike the BglG protein, the MS2 CP showed a strong tendency to aggregate when its concentration in the cytoplasm was increased by deletion of its NLS. This tendency to aggregate may be increased by a local MS2 CP accumulation within the PD cavities, thereby provoking local entrapment of mRNA in an mRNA-MS2 CP aggregate. In addition, both RBPs must dimerize to recognize and bind to their respective RNA motifs (Wu et al., 2012; Amster-Choder and Wright, 1992). The MS2 system thereby involves a $90 \mathrm{kDa}$ complex placed at each SL position of the tagged mRNA. The size of this complex is larger than the BglG complex (60 $\mathrm{kDa}$ ) and could impose steric hindrances for the passage through the pore. Cell-to-cell transport of mRNA without accumulation in the transport channel is consistent with mRNA transport through the nuclear pore complex in animal cells, in which the passage through the pore is not the rate limiting step despite the size of the packed mRNA-protein complex (Grünwald and Singer, 2010; Mor et al., 2010).

While MP mRNA was always associated with MP, not all localizations of MP were associated with the presence of MP mRNA. Importantly, MP mRNA was localized to mobile MP particles but not to MP accumulating along MTs. Given that MP binds RNA in vitro in a non-sequencespecific manner (Citovsky et al., 1990), MP may bind any RNA in its vicinity, thus most likely 
its own coding RNA upon translation in association with the ER. Thus, the absence of MP mRNA from MP-associated MTs could be explained by a specific mechanism that traps the mRNA into MP particles during translation. However, the absence of MP mRNA from MPassociated MT may also indicate that the MP fractions associated with particles and MTs differ in RNA-binding activity. For example, the two MP fractions could be associated with different host factors or be differentially modified by posttranslational modifications. Support for this hypothesis is provided by the previous finding that MP becomes ubiquitinated during infection (Reichel and Beachy, 2000), except for MP associated with MT (Ashby et al., 2006). MP is also a target for phosphorylation (Waigmann, 2000; Citovsky et al., 1993; Haley et al., 1995; Kawakami et al., 2003; Watanabe et al., 1992) and may therefore be differentially phosphorylated when associated with MT.

We believe that the BgIG RNA labeling method adapted to plants in this work will facilitate the dissection of pathways involved in RNA transport and localization in plants. With respect to TMV and other viruses, this tool may allow the identification of cellular factors associated with MP particles and viral RNA movement. Importantly, we show that the BglG system can be used to visualize the cell-to-cell transport of in vivo expressed mRNA and, therefore, represents a fundamental tool to characterize RNA elements required for the transport of mobile transcripts.

\section{Methods}

\section{Plasmid constructs}

Constructs for expression of MP:RFP-SL (pMDC32-MP:mRFP-SL) and NLS:MS2CP:GFP (pNLS:MS2CP:GFP) where previously described (Sambade et al., 2008). The construct for expression of MP:RFP-BoxB was created by Gateway cloning using the entry vector pZeo- 
MP:mRFP (Sambade et al., 2008) and pSCJ215 (Schönberger et al., 2012) as destination vector. The binary plasmid for expression of $\lambda N$ :eGFP:NLS was described elsewhere (Schönberger et al., 2012). BgIG:mCherry:NLS was PCR-amplified from pBgIG-mCherry (Chen et al., 2009) using primers pBglG:mCh:NLSfw (5'ggggacaagtttgtacaaaaaagcaggcttaatgaacatgcaaatcacc-3') and pBglG:mCh:NLSrev (5'ggggaccactttgtacaagaaagctgggtatacctttctcttctttttggc-3'). The PCR product was cloned into $\mathrm{pDONR}^{\mathrm{TM}} /$ Zeo using a BP clonase reaction (Thermo Fisher Scientific). The resulting entry vector pZeo-BglG:mCherry:NLS was confirmed by Sanger DNA sequencing and then used in an LR recombination reaction with the destination vector pMDC32 (Curtis and Grossniklaus, 2003) to obtain the binary expression vector pBglG:mCherry:NLS. A tandem of 18 BgIG-SLs was PCR amplified from pBgIG-SL (Chen et al., 2009) using primers BglGSLfw (5'- ggggacaagtttgtacaaaaaagcaggcttatttaattaatgcagaattcgcccttgg-3') and BgIG-SLrev (5'- ggggaccactttgtacaagaaagctgggtagagctcaattcgcccttaggtcgc-3'). The PCR product was cloned into $\mathrm{pDONR}{ }^{\mathrm{TM}} /$ Zeo by a BP clonase reaction. The resulting vector was confirmed by Sanger DNA sequencing and digested with $\mathrm{Pacl} / \mathrm{Sacl}$ restriction endonucleases (NewEngland Biolabs, MA USA). The released fragment was separated by electrophoresis, purified from the agarose gel and ligated by T4-DNA ligase into Pacl/Sacl digested pMDC32 destination vector (Curtis and Grossniklaus, 2003) to obtain pMDC32-BgIG-SLs. Similarly, the coding region of MP:GFP was PCR-amplified from pTMV-MP:GFP5 (Boyko et al., 2000c) using primers MPGFPfw (5'- ggggacaagtttgtacaaaaaagcaggcttaatggctctagttgttaaa3') and MPGFPrev (5'- ggggaccactttgtacaagaaagctgggtatttagcggccgcgactagttca-3'). The product was cloned into pDONR ${ }^{\mathrm{TM}} /$ Zeo by a BP clonase reaction (Thermo Fisher Scientific). The resulting entry clone was confirmed by Sanger DNA sequencing and used in an LR recombination together with either pMDC32 or pMDC32-BgIG-SL to obtain the binary vectors pMP:GFP or pMP:GFP-SL. To express the different RBPs without NLS, the RBPencoding sequences were amplified by PCR using MS2CPfw (5'- 
ggggacaagtttgtacaaaaaagcaggcttaatggcttctaactttact-3') and MS2CPrev (5'ggggaccactttgtacaagaaagctgggtagtagatgccggagtttgc-3') for MS2 CP, primers $\lambda N f w$ (5'ggggacaagtttgtacaaaaaagcaggcttaatggccagatctgacgcc -3 ') and $\lambda \mathrm{Nrev}$ (5'ggggaccactttgtacaagaaagctgggtaaccgttggcggccttcca-3') for $\lambda \mathrm{N}$, and BglGfw (5'ggggacaagtttgtacaaaaaagcaggcttaatgaacatgcaaatcacc-3') and BglGrev (5'ggggaccactttgtacaagaaagctgggtaggtggcgaccggtagcaa-3') for BgIG. The PCR products were cloned into $\mathrm{pDONR}{ }^{\mathrm{TM}} / \mathrm{Zeo}$, confirmed by Sanger DNA sequencing, and used for recombination with either pB7FWG2 or pH7RWG2 (Karimi et al., 2002) using LR clonase to obtain the C-terminal fusion to eGFP (pB7-MS2CP:eGFP and $p B 7-\lambda N$ :eGFP) or mRFP (pH7-BglG:mRFP). All plasmids were selected and propagated in E. coli (Top 10 strain, Invitrogen) and transferred by electroporation into Agrobacterium tumefaciens (strain GV3101).

\section{Protein expression in plants.}

Cultures were grown over night at $28^{\circ} \mathrm{C}$ and at $180-200 \mathrm{rpm}$ agitation on LB medium under appropriate antibiotic selection. Rifampicin $(50 \mu \mathrm{g} / \mathrm{ml})$ and gentamicin $(50 \mu \mathrm{g} / \mathrm{ml})$ for all the constructs expressed from GV3101 A. tumefaciens strain, kanamycin $(50 \mu \mathrm{g} / \mathrm{ml})$ for bacteria containing pSCJ (for the expression of $\lambda \mathrm{N}$ :eGFP:NLS and MP:RFP-BoxB) and pMDC based vectors or Spectinomicin $(100 \mu \mathrm{g} / \mathrm{ml})$ for $\mathrm{pB} 7$ or $\mathrm{pH} 7$ based vectors (for the expression of NLS:MS2CP:eGFP and all the non NLS tagged versions of the RBPs). Cultures were harvested by centrifugation during $5 \mathrm{~min}$ at $5.000 \mathrm{x}$ g, resuspended in sterile distilled water and diluted to specific optical densities $\left(\mathrm{OD}_{600}\right)$ before agroinfiltration. For experiments shown in Fig. 1 and Fig. 2, cultures with an $\mathrm{OD}_{600}$ of 0.4 were used. For experiments in Fig. 3, MP- and RBP-encoding bacteria were used with an $\mathrm{OD}_{600}$ of 0.4 and 0.1 , respectively. For expression of BgIG:mRFP in isolated cells (Fig. 4), the bacteria were diluted to an $\mathrm{OD}_{600}$ of $1,0 \times 10^{-4}$ whereas the MP:GFP-encoding cultures were used with an $\mathrm{OD}_{600}$ of 0.4 to 
ensure expression throughout the infiltrated tissue. Leaves were co-infiltrated with agrobacteria containing pBin61-P19 (kanamycin resistance) (Silhavy et al., 2002) for coexpression of the tombusvirus protein $\mathrm{P} 19\left(\mathrm{OD}_{600}=0.2\right)$ to inhibit post-transcriptional RNA silencing and ensure efficient expression of the constructs. Cultures were infiltrated into the abaxial side of fully expanded leaves of 4-5 weeks old Nicotiana benthamiana plants using $2.0 \mathrm{ml}$ syringes without needles. For all RNA labeling experiments, one-half of the leaf was used for the expression of the SL-tagged mRNA while the other half was used for the corresponding non-tagged control. Plants were grown and maintained in growth chambers, with $16 \mathrm{~h}, 24^{\circ} \mathrm{C} / 8 \mathrm{~h}, 22^{\circ} \mathrm{C}$ day/night cycles.

Transgenic $N$. benthamiana plants were generated by leaf disk transformation (Horsch et al., 1985) using $A$. tumefaciens GV3101 bacteria carrying the plasmid pH7-BglG:mRFP.

\section{Fluorescence microscopy and image processing}

For analysis of leaf tissues by fluorescence microscopy, leaf discs were excised and mounted on microscope slides and vacuum-infiltrated with water. The leaf discs were excised at 48-72 hours post infiltration (hpi) for experiments shown in Figure 1 and Figure 2, at 36-48 hpi for experiments shown in Figure 3, and at 96 hpi for experiments shown in Figure 4. Abaxial epidermal cells were imaged using a Zeiss LSM780 confocal microscope using excitation/emission wavelengths of $488 / 505-545$ and $543 / 585-615$ for the imaging of GFP and RFP/mCherry, respectively. Images were processed using ImageJ (version 1.51j8) software. Particles were tracked in movies using the ImageJ macro "OverlayCrosses" (https://git.io/fjnDF).

\section{Immunoprecipitation and protein analysis}

40 hs after infiltration, $0,5 \mathrm{~g}$ of tissue belonging to leaf material expressing either MP:GFP or MP:GFP-SL together with BgIG:mRFP were ground to fine powder in liquid N2 and resuspended in $1.0 \mathrm{ml}$ of ice-cold extraction buffer $(20 \mathrm{mM}$ Tris/HCL pH 7.5, $150 \mathrm{mM} \mathrm{NaCl}$, 
$0.5 \mathrm{mM}$ EDTA, $0.5 \%$ NP40, 1 x Complete Protease Inhibitor (PI, Roche)). The lysate was cleared by 5 min centrifugation at $20.000 \times \mathrm{g}$ at $4{ }^{\circ} \mathrm{C}$, diluted with one volume of ice-cold dilution buffer (20 mM Tris/HCL pH 7.5, $150 \mathrm{mM} \mathrm{NaCl,} 0.5 \mathrm{mM}$ EDTA 1 x PI), and an aliquot reserved for immunoblot analysis (input fraction, IN). Cleared and diluted protein extract was incubated with $20 \mu \mathrm{l}$ of GFP-Trap agarose beads (Chromotek, Germany) for $1.5 \mathrm{~h}$ at $4{ }^{\circ} \mathrm{C}$ with gentle end-over-end agitation. Beads were collected by centrifugation at $2500 \times \mathrm{g}$ for 2 min at $4{ }^{\circ} \mathrm{C}$. The supernatant was placed aside for immunoblot analysis (flow-through fraction, FT) and beads were washed two times with washing buffer (20 mM Tris/HCL pH 7.5, $150 \mathrm{mM} \mathrm{NaCl}, 0.5 \mathrm{mM}$ EDTA). Beads were resuspended in $50 \mu \mathrm{l}$ of $2 \mathrm{X}$ Laemmli buffer, boiled for $10 \mathrm{~min}$ and collected by centrifugation at $2500 \mathrm{xg}$ for $2 \mathrm{~min}$ at $4{ }^{\circ} \mathrm{C}$, and proteins in the supernatant were collected and used for immunoblot analysis (immunoprecipitated fraction, IP). IN, FT and IP protein samples were separated by $12 \%$ SDS-PAGE and blotted onto PVDF membrane for immunoblot detection. RFP-fused proteins were detected with anti-RFP 6 G6 mouse monoclonal antibody (Chromotek, Germany), and the TMV MP was detected using MP antibodies raised in rabbit (Boyko et al., 2000c). Horseradish peroxidase conjugated anti-mouse (BioRad, USA) and anti-rabbit (Invitrogen) were used as secondary antibodies. Chemiluminescent reagent was used for detection of peroxidase activity according to the manufacturer's instructions (GE, ECL Plus Western Blotting Detection Reagents, UK). Chemiluminescent signal intensities of immunoreactive bands were determined within digital images using ImageJ (version 1.51j8) software. Average pixel intensities within specific regions of interest (ROI) applied to bands of samples containing BgIG:SL-tagged mRNA were corrected by the average pixel intensities measured within the same ROI size above or below the measured bands (background). The same procedure was performed with ROls of the same size applied to corresponding bands of samples containing non-tagged mRNA. Results are displayed as the ratio between the corrected average pixel intensities of both samples. 


\section{Statistical analysis}

Intensity values of BglG:mRFP immunoreactive bands shown in Figure 3 were compared with an unpaired t-test using GraphPad prism (V 6.01).

The statistical probability for the independent transformation of adjacent cells by diluted agrobacteria was calculated by Poisson statistics expressed as $P(x)=\left(e^{-\mu} \mu^{x}\right) / x$ !, where "x" is the number of these occurrences [number of independently transformed cells (B) found adjacent to initially transformed cells $(A)]$ and " $\mu$ " is the ratio between adjacent cells concerned $(C)$ divided by the number of all cells (D) subtracted by the sum of initially transformed cells (A) and adjacent cells (C). Leaf disks analyzed (9,0 mm diameter) in the experiments described in Figure 4 contained approximately 1400 epidermal cells (abaxial side) and each cell had four to seven adjacent cells. MP:GFP-SL-expressing leaf discs transformed with diluted agrobacteria to express BglG:mRFP in isolated cells led to the occurrence of 28 red fluorescent, BglG:mRFP-expressing spots of cells on the average. Nine of these spots consisted of more than one fluorescent epidermal cell (see Table S1). According to the Poisson distribution, the likelihood in a tissue of 1400 cells that nine out of $(9+28=) 37$ transformation events occur in adjacent cells is extremely unlikely. Considering that each epidermal cell has four adjacent epidermal cells, the 28 initially transformed cells (A) have 112 adjacent cells (C). Given that and the number of all cells (D) is 1400 , the number of cells that are initially transformed $(\mathrm{A})$ is 28 and the number of neighboring cells concerned $(C)$ is 112 , the probability to obtain nine independent transformation events $(B)$ adjacent to 28 initially transformed cells $(A)$ is $P(9)=\left(e^{-(112 / 1260)} \times(112 / 1260)^{9}\right) / 9 !=8,73 \times$ $10^{-16}$. Under the assumption that each cell has seven adjacent the probability is $P(9)=\left(e^{-}\right.$ $\left.(196 / 1176) \times(196 / 1176)^{9}\right) / 9 !=2,3 \times 10^{-13}$. 


\section{Data availability statement}

All relevant data can be found within the manuscript and its supporting materials. A documentation of the three replicate immunoprecipitation experiments used for quantification shown in Fig. $3 f$ is available at https://doi.org/10.5281/zenodo.4047215.

\section{Acknowledgements}

We thank Thomas Dresselhaus (Cell Biology and Plant Biochemistry, University of Regensburg, Germany) for providing plasmids pSCJ215 and pSCJ206 and Wei-Shau Hu (HIV Drug Resistance Program, NCI Frederick, USA) for providing plasmids pBgIG-SL and pBgIG:mCherry. We thank Jérôme Mutterer (IBMP-CNRS, Strasbourg, France) for support in preparing movies and Claudia Ruscitti (Departamento de Matemática, Facultad de Ciencias Exactas-UNLP, Argentina) for help in the statistical analysis of mRNA cell-to-cell transport. This work was performed with financial support from the ANR (grant ANR-08BLAN-0244-02) to MH, CONICET (grant PIP 2015-0847) to EJP, and CNRS-CONICET (PICS 2014) to $\mathrm{MH}$ and EJP.

\section{Author contributions}

EJP and $\mathrm{MH}$ conceived the idea and designed the study, EJP and GRL performed the experiments, EJP, GRL and $\mathrm{MH}$ analyzed the results, EJP and $\mathrm{MH}$ wrote the manuscript. All authors approved the manuscript prior to publication.

\section{Conflicts of interest}

The authors declare no conflicts of interest. 


\section{Supporting Information}

Figure S1. Motility of RNA-containing MP:GFP particles.

Figure S2. Analysis of BgIG:mRFP recruitment by SL-tagged MP:GFP mRNA.

Figure S3. MP:GFP particle motility is not influenced by BglG:mRFP binding.

Figure S4. Imaging of MP mRNA in BgIG:mRFP transgenic plants.

Table S1. MP:GFP mRNA cell-to-cell movement assay.

Movie S1. MP:RFP mRNA imaging using MS2 system.

Movie S2. MP:RFP mRNA imaging using $\lambda \mathrm{N}$ system.

Movie S3. MP:GFP mRNA imaging using BglG system.

Movie S4. Accumulation pattern of MS2CP:eGFP.

Movie S5. Accumulation pattern of $\lambda$ N:eGFP.

Movie S6. Accumulation pattern of BglG:mRFP.

Movie S7. BglG:mRFP localizes independently of MP:GFP.

Movie S8. BgIG:mRFP-mediated detection of tagged MP:GFP mRNA in MP:GFP particles.

\section{References:}

Amari, K., Donato, M. Di, Dolja, V. V. and Heinlein, M. (2014) Myosins VIII and XI play distinct roles in reproduction and transport of tobacco mosaic virus. PLoS Pathog., 10, e1004448. 
Amster-Choder, O. and Wright, A. (1992) Modulation of the dimerization of a transcriptional antiterminator protein by phosphorylation. Science, 257, 1395-8.

Ashby, J., Boutant, E., Seemanpillai, M., Sambade, A., Ritzenthaler, C. and Heinlein, M. (2006) Tobacco mosaic virus movement protein functions as a structural microtubule-associated protein. J. Virol., 80, 8329-8344.

Bertrand, E., Chartrand, P., Schaefer, M., Shenoy, S.M., Singer, R.H. and Long, R.M. (1998) Localization of ASH1 mRNA particles in living yeast. Mol. Cell, 2, 437-445.

Borniego, M.B., Karlin, D., Peña, E.J., Robles Luna, G. and García, M.L. (2016) Bioinformatic and mutational analysis of ophiovirus movement proteins, belonging to the 30K superfamily. Virology, 498, 172-180.

Boyko, V., Ashby, J.A., Suslova, E., Ferralli, J., Sterthaus, O., Deom, C.M. and Heinlein, M. (2002) Intramolecular complementing mutations in tobacco mosaic virus movement protein confirm a role for microtubule association in viral RNA transport. J. Virol., 76, 3974-3980.

Boyko, V., Ferralli, J., Ashby, J., Schellenbaum, P. and Heinlein, M. (2000a) Function of microtubules in intercellular transport of plant virus RNA. Nat. Cell Biol., 2, 826-832.

Boyko, V., Ferralli, J. and Heinlein, M. (2000b) Cell-to-cell movement of TMV RNA is temperature-dependent and corresponds to the association of movement protein with microtubules. Plant J., 22, 315-325.

Boyko, V., Hu, Q., Seemanpillai, M., Ashby, J. and Heinlein, M. (2007) Validation of microtubule-associated tobacco mosaic virus RNA movement and involvement of microtubule-aligned particle trafficking. Plant J., 51, 589-603.

Boyko, V., Laak, J. van der, Ferralli, J., Suslova, E., Kwon, M.O. and Heinlein, M. 
(2000c) Cellular targets of functional and dysfunctional mutants of tobacco mosaic virus movement protein fused to green fluorescent protein. J. Virol., 74, 11339-46.

Brandner, K., Sambade, A., Boutant, E., Didier, P., Mely, Y., Ritzenthaler, C. and Heinlein, M. (2008) Tobacco mosaic virus movement protein interacts with green fluorescent protein-tagged microtubule end-binding protein 1. Plant Physiol., 147, 611623.

Brunkard, J.O., Runkel, A.M. and Zambryski, P.C. (2015) The cytosol must flow: Intercellular transport through plasmodesmata. Curr. Opin. Cell Biol., 35, 13-20.

Buxbaum, A.R., Haimovich, G. and Singer, R.H. (2015) In the right place at the right time: Visualizing and understanding mRNA localization. Nat. Rev. Mol. Cell Biol., 16, 95109.

Chen, J., Nikolaitchik, O., Singh, J., et al. (2009) High efficiency of HIV-1 genomic RNA packaging and heterozygote formation revealed by single virion analysis. Proc. Natl. Acad. Sci. U. S. A., 106, 13535-40.

Chin, A. and Lécuyer, E. (2017) RNA localization: Making its way to the center stage. Biochim. Biophys. Acta. Gen. Subj., 1861, 2956-2970.

Christensen, N.M., Oparka, K.J. and Tilsner, J. (2010) Advances in imaging RNA in plants. Trends Plant Sci., 15, 196-203.

Citovsky, V., Knorr, D., Schuster, G. and Zambryski, P. (1990) The P30 movement protein of tobacco mosaic virus is a single-strand nucleic acid binding protein. Cell, 60, $637-647$.

Citovsky, V., McLean, B.G., Zupan, J.R. and Zambryski, P. (1993) Phosphorylation of tobacco mosaic virus cell-to-cell movement protein by a developmentally regulated 
plant cell wall-associated protein kinase. Genes Dev., 7, 904-10.

Curtis, M.D. and Grossniklaus, U. (2003) A gateway cloning vector set for high-throughput functional analysis of genes in planta. Plant Physiol., 133, 462-469.

Daigle, N. and Ellenberg, J. (2007) AN-GFP: An RNA reporter system for live-cell imaging. Nat. Methods, 4, 633-636.

Eliscovich, C. and Singer, R.H. (2017) RNP transport in cell biology: the long and winding road. Curr. Opin. Cell Biol., 45, 38-46.

Ferralli, J., Ashby, J., Fasler, M., Boyko, V. and Heinlein, M. (2006) Disruption of microtubule organization and centrosome function by expression of tobacco mosaic virus movement protein. J. Virol., 80, 5807-5821.

George, L., Indig, F.E., Abdelmohsen, K. and Gorospe, M. (2018) Intracellular RNAtracking methods. Open Biol., 8, 180104.

Goelet, P., Lomonossoff, G.P., Butler, P.J., Akam, M.E., Gait, M.J. and Karn, J. (1982) Nucleotide sequence of tobacco mosaic virus RNA. Proc. Natl. Acad. Sci. U. S. A., 79, 5818-5822.

Grünwald, D. and Singer, R.H. (2010) In vivo imaging of labelled endogenous $\beta$-actin mRNA during nucleocytoplasmic transport. Nature, 467, 604-7.

Haley, A., Hunter, T., Kiberstis, P. and Zimmern, D. (1995) Multiple serine phosphorylation sites on the $30 \mathrm{kDa}$ TMV cell-to-cell movement protein synthesized in tobacco protoplasts. Plant J., 8, 715-24.

Ham, B.-K. and Lucas, W.J. (2017) Phloem-mobile RNAs as systemic signaling agents. Annu. Rev. Plant Biol., 68, 173-195.

Hamada, S., Ishiyama, K., Choi, S.-B., Wang, C., Singh, S., Kawai, N., Franceschi, V.R. 
and Okita, T.W. (2003) The transport of prolamine RNAs to prolamine protein bodies in living rice endosperm cells. Plant Cell, 15, 2253-2264.

Heinlein, M. (2015) Plant virus replication and movement. Virology, 479-480, 657-671.

Heinlein, M. (2002) Plasmodesmata: Dynamic regulation and role in macromolecular cellto-cell signaling. Curr. Opin. Plant Biol., 5, 543-552.

Heinlein, M., Epel, B.L., Padgett, H.S. and Beachy, R.N. (1995) Interaction of tobamovirus movement proteins with the plant cytoskeleton. Science, 270, 1983-1985.

Heinlein, M., Padgett, H.S., Gens, J.S., Pickard, B.G., Casper, S.J., Epel, B.L. and Beachy, R.N. (1998) Changing patterns of localization of the tobacco mosaic virus movement protein and replicase to the endoplasmic reticulum and microtubules during infection. Plant Cell, 10, 1107-1120.

Holt, C.A. and Beachy, R.N. (1991) In vivo complementation of infectious transcripts from mutant tobacco mosaic virus cDNAs in transgenic plants. Virology, 181, 109-117.

Holt, C.E. and Bullock, S.L. (2009) Subcellular mRNA localization in animal cells and why it matters. Science, 326, 1212-1216.

Horsch, R.B., Fry, J.E., Hoffmann, N.L., Eichholtz, D., Rogers, S.G. and Fraley, R.T. (1985) A simple and general method for transferring genes into plants. Science, 227, $1229-1231$.

Ishikawa, K., Tamura, K., Fukao, Y. and Shimada, T. (2020) Structural and functional relationships between plasmodesmata and plant endoplasmic reticulum-plasma membrane contact sites consisting of three synaptotagmins. New Phytol., 226, 798808.

Jeffery, W.R., Tomlinson, C.R. and Brodeur, R.D. (1983) Localization of actin messenger 
RNA during early ascidian development. Dev. Biol., 99, 408-17.

Kapila, J., Rycke, R. De, Montagu, M. Van and Angenon, G. (1997) An Agrobacteriummediated transient gene expression system for intact leaves. Plant Sci., 122, 101-108.

Karimi, M., Inze, D. and Depicker, A. (2002) GATEWAYTM vectors for Agrobacteriummediated plant transformation. Trends Plant Sci., 7, 193-195.

Kawakami, S., Hori, K., Hosokawa, D., Okada, Y. and Watanabe, Y. (2003) Defective tobamovirus movement protein lacking wild-type phosphorylation sites can be complemented by substitutions found in revertants. J. Virol., 77, 1452-61.

Kehr, J. and Buhtz, A. (2008) Long distance transport and movement of RNA through the phloem. J. Exp. Bot., 59, 85-92.

Kehr, J. and Kragler, F. (2018) Long distance RNA movement. New Phytol., 218, 29-40.

Larson, D.R., Zenklusen, D., Wu, B., Chao, J.A. and Singer, R.H. (2011) Real-time observation of transcription initiation and elongation on an endogenous yeast gene. Science, 332, 475-8.

Lécuyer, E., Yoshida, H., Parthasarathy, N., Alm, C., Babak, T., Cerovina, T., Hughes, T.R., Tomancak, P. and Krause, H.M. (2007) Global analysis of mRNA localization reveals a prominent role in organizing cellular architecture and function. Cell, 131, 174187.

Levy, A., Zheng, J.Y. and Lazarowitz, S.G. (2015) Synaptotagmin SYTA forms ER-plasma membrane junctions that are recruited to plasmodesmata for plant virus movement. Curr. Biol., 25, 2018-25.

Lewis, J.D. and Lazarowitz, S.G. (2010) Arabidopsis synaptotagmin SYTA regulates endocytosis and virus movement protein cell-to-cell transport. Proc. Natl. Acad. Sci., 
107, 2491-2496.

Liu, L. and Chen, X. (2018) Intercellular and systemic trafficking of RNAs in plants. Nat. Plants, 4, 869-878.

Lucas, W.J. (2006) Plant viral movement proteins: Agents for cell-to-cell trafficking of viral genomes. Virology, 344, 169-184.

Lucas, W.J., Bouché-Pillon, S., Jackson, D.P., Nguyen, L., Baker, L., Ding, B. and Hake, S. (1995) Selective trafficking of KNOTTED1 homeodomain protein and its mRNA through plasmodesmata. Science, 270, 1980-3.

Lucas, W.J. and Lee, J.Y. (2004) Plasmodesmata as a supracellular control network in plants. Nat. Rev. Mol. Cell Biol., 5, 712-726.

Luo, K.-R., Huang, N.-C. and Yu, T.-S. (2018) Selective targeting of mobile mRNAs to plasmodesmata for cell-to-cell movement. Plant Physiol., 177, 604-614.

Martin, K.C. and Ephrussi, A. (2009) mRNA localization: gene expression in the spatial dimension. Cell, 136, 719-730.

Michaud, M., Ubrig, E., Filleur, S., Erhardt, M., Ephritikhine, G., Marechal-Drouard, L. and Duchene, A.-M. (2014) Differential targeting of VDAC3 mRNA isoforms influences mitochondria morphology. Proc. Natl. Acad. Sci., 111, 8991-8996.

Mofatteh, M. and Bullock, S.L. (2017) SnapShot: Subcellular mRNA localization. Cell, 169, $178-178$.

Mor, A., Suliman, S., Ben-Yishay, R., Yunger, S., Brody, Y. and Shav-Tal, Y. (2010) Dynamics of single mRNP nucleocytoplasmic transport and export through the nuclear pore in living cells. Nat. Cell Biol., 12, 543-552.

Nazim Uddin, M. and Kim, J.Y. (2013) Intercellular and systemic spread of RNA and RNAi 
in plants. Wiley Interdiscip. Rev. RNA, 4, 279-293.

Nebenführ, A., Gallagher, L.A., Dunahay, T.G., Frohlick, J.A., Mazurkiewicz, A.M., MeehI, J.B. and Staehelin, L.A. (1999) Stop-and-go movements of plant Golgi stacks are mediated by the acto-myosin system. Plant Physiol., 121, 1127-42.

Niehl, A., Peña, E.J., Amari, K. and Heinlein, M. (2013) Microtubules in viral replication and transport. Plant J., 75, 290-308.

Okita, T.W. and Choi, S.B. (2002) mRNA localization in plants: Targeting to the cell's cortical region and beyond. Curr. Opin. Plant Biol., 5, 553-559.

Ouko, M.O., Sambade, A., Brandner, K., Niehl, A., Peña, E., Ahad, A., Heinlein, M. and Nick, P. (2010) Tobacco mutants with reduced microtubule dynamics are less susceptible to TMV. Plant J., 62, 829-839.

Peiro, A., Martinez-Gil, L., Tamborero, S., Pallas, V., Sanchez-Navarro, J.A., Mingarro, I. and Simon, A. (2014) The tobacco mosaic virus movement protein associates with but does not integrate into biological membranes. J. Virol., 88, 3016-3026.

Peña, E.J. and Heinlein, M. (2013) Cortical microtubule-associated ER sites: Organization centers of cell polarity and communication. Curr. Opin. Plant Biol., 16, 764-773.

Peña, E.J. and Heinlein, M. (2012) RNA transport during TMV cell-to-cell movement. Front. Plant Sci., 3, 1-10.

Pitzalis, N. and Heinlein, M. (2017) The roles of membranes and associated cytoskeleton in plant virus replication and cell-to-cell movement. J. Exp. Bot., 69, 117-132.

Reagan, B.C., Ganusova, E.E., Fernandez, J.C., McCray, T.N. and Burch-Smith, T.M. (2018) RNA on the move: The plasmodesmata perspective. Plant Sci., 275, 1-10.

Reichel, C. and Beachy, R.N. (2000) Degradation of tobacco mosaic virus movement 
protein by the 26 S proteasome. J. Virol., 74, 3330-3337.

Robles Luna, G., Peña, E.J., Borniego, M.B., Heinlein, M. and Garcia, M.L. (2013) Ophioviruses CPsV and MiLBVV movement protein is encoded in RNA 2 and interacts with the coat protein. Virology, 441, 152-161.

Ryder, P. V. and Lerit, D.A. (2018) RNA localization regulates diverse and dynamic cellular processes. Traffic, 19, 496-502.

Sambade, A., Brandner, K., Hofmann, C., Seemanpillai, M., Mutterer, J. and Heinlein, M. (2008) Transport of TMV movement protein particles associated with the targeting of RNA to plasmodesmata. Traffic, 9, 2073-2088.

Scholthof, K.-B.G., Adkins, S., Czosnek, H., et al. (2011) Top 10 plant viruses in molecular plant pathology. Mol. Plant Pathol., 12, 938-54.

Schönberger, J., Hammes, U.Z. and Dresselhaus, T. (2012) In vivo visualization of RNA in plants cells using the $\lambda n 22$ system and a GATEWAY-compatible vector series for candidate RNAs. Plant J., 71, 173-181.

Silhavy, D., Molnár, A., Lucioli, A., Szittya, G., Hornyik, C., Tavazza, M. and Burgyán, J. (2002) A viral protein suppresses RNA silencing and binds silencing-generated, 21to 25-nucleotide double-stranded RNAs. EMBO J., 21, 3070-80.

Thieme, C.J., Rojas-Triana, M., Stecyk, E., et al. (2015) Endogenous Arabidopsis messenger RNAs transported to distant tissues. Nat. Plants, 1, 1-8.

Tian, L., Chou, H.L., Fukuda, M., Kumamaru, T. and Okita, T.W. (2020) mRNA localization in plant cells. Plant Physiol., 182, 97-109.

Tilsner, J. (2015) Techniques for RNA in vivo imaging in plants. J. Microsc., 258, 1-5.

Tutucci, E., Vera, M., Biswas, J., Garcia, J., Parker, R. and Singer, R.H. (2018) An 
improved MS2 system for accurate reporting of the mRNA life cycle. Nat. Methods, 15, 81-89.

Vera, M., Biswas, J., Senecal, A., Singer, R.H. and Park, H.Y. (2016) Single-cell and single-molecule analysis of gene expression regulation. Annu. Rev. Genet., 50, 267291.

Waigmann, E. (2000) Regulation of plasmodesmal transport by phosphorylation of tobacco mosaic virus cell-to-cell movement protein. EMBO J., 19, 4875-4884.

Watanabe, Y., Ogawa, T. and Okada, Y. (1992) In vivo phosphorylation of the 30-kDa protein of tobacco mosaic virus. FEBS Lett., 313, 181-4.

Wilkie, G.S. and Davis, I. (2001) Drosophila wingless and pair-rule transcripts localize apically by dynein-mediated transport of RNA particles. Cell, 105, 209-219.

Wu, B., Chao, J.A. and Singer, R.H. (2012) Fluorescence fluctuation spectroscopy enables quantitative imaging of single mRNAs in living cells. Biophys. J., 102, 2936-2944.

Wu, B., Eliscovich, C., Yoon, Y.J. and Singer, R.H. (2016) Translation dynamics of single mRNAs in live cells and neurons. Science, 352, 1430-5.

Yang, L., Perrera, V., Saplaoura, E., et al. (2019) m5C methylation guides systemic transport of messenger RNA over graft junctions in plants. Curr. Biol., 29, 24652476.e5.

Yang, Y., Mao, L., Jittayasothorn, Y., Kang, Y., Jiao, C. and Fei, Z. (2015) Messenger RNA exchange between scions and rootstocks in grafted grapevines. BMC Plant Biol., $1-14$.

Yuan, C., Lazarowitz, S.G. and Citovsky, V. (2018) The plasmodesmal localization signal of TMV MP is recognized by plant synaptotagmin SYTA. MBio, 9, 1-8. 
Zambryski, P. and Crawford, K. (2000) Plasmodesmata: Gatekeepers for cell-to-cell transport of developmental signals in plants. Annu. Rev. Cell Dev. Biol., 16, 393-421.

Zhang, Z., Zheng, Y., Ham, B.-K., Chen, J., Yoshida, A., Kochian, L. V, Fei, Z. and Lucas, W.J. (2016) Vascular-mediated signalling involved in early phosphate stress response in plants. Nat. Plants, 2, 16033. 


\section{FIGURE LEGENDS}

\section{Figure 1. Systems used for the in vivo imaging of TMV MP mRNA.}

a, MS2 system. Top, representation of transcripts (blue line) and encoded proteins (boxes). Middle, cortical view of a $N$. benthamiana leaf epidermal cell showing NLS:MS2CP:GFP-labeled MP:RFP mRNA (green channel, left), MP:RFP (red channel, center) and the colocalization of the RNA and protein (yellowish signal in the merged figure, right). The colocalization in MP particles is indicated by arrowheads. Bottom, central view of a cell from the same experiment showing NLS:MS2CP:GFPlabeled MP:RFP mRNA (left) and MP:RFP (center) colocalizing at PD (right; examples of PD localization are indicated by arrows). $\mathbf{b}, \lambda \mathrm{N}$ system. Top, representation of transcripts (blue line) and encoded protein (boxes). Middle, cortical view of a $N$. benthamiana epidermal cell showing $\lambda \mathrm{N}$ :eGFP:NLS-labeled MP:RFP mRNA (green channel, left), MP:RFP (red channel, center), and colocalization of the RNA and protein (yellow signal in the merged channel, right). Examples of colocalization in MP particles are indicated by arrowheads. Bottom, central view of a cell from the same experiment showing the localization of $\lambda \mathrm{N}$ :eGFP:NLS-labeled MP:RFP mRNA (green channel, left), MP:RFP (red channel, center), and colocalization in the merged image (yellowish signal, right; examples of PD localization are indicated by arrows). c, BglG system. Top, representation of transcripts (blue line) and encoded proteins (boxes). Middle, cortical section of a $N$. benthamiana epidermal cell showing MP:GFP-tagged mobile particles (green, left), BglG:mCherry:NLS-labeled MP:GFP mRNA (red, center) and colocalization of MP:GFP and its mRNA in the merged image (yellow signal, right). Examples of colocalization in MP particles are indicated by arrowheads. Bottom, central view of a cell from the same experiment showing MP:GFP (green, left), BgIG:mCherry:NLSlabeled MP:GFP mRNA (red, center), and the colocalization of MP:GFP mRNA and protein in the merged image (yellow signal, right). Examples of PD localization are indicated by arrows. NLS, nuclear localization signal; SL, stem loops; Scale bars, $5 \mu \mathrm{m}$. 
Figure 2. Accumulation patterns of the different RBPs expressed without a NLS. a, Central views of $N$. benthamiana epidermal cells expressing MS2CP:eGFP (left), $\lambda N$ :eGFP (middle), or BglG:mRFP (right). Scale bar, $50 \mu \mathrm{m}$. b, Cortical views of $N$. benthamiana epidermal cells expressing MS2CP:eGFP (left), $\lambda \mathrm{N}$ :eGFP (middle), or BglG:mRFP (right). Scale bars, $5 \mu \mathrm{m}$.

Figure 3. Detection of MP:GFP mRNA using the BgIG system. a, cortical view of a $N$. benthamiana epidermal cell expressing MP:GFP (green channel, left) and BgIG:mRFP (red channel, center). The merged image is shown on the right. $\mathbf{b}$, cortical view of a $N$. benthamiana epidermal cell expressing MP:GFP from mRNA tagged with BgIG-SLs (MP:GFP-SL, green channel, left) together with BgIG:mRFP (red channel, middle). The merged image shows co-localization of MP:GFP mRNA and protein (yellow signal, right). c, central view of a $N$. benthamiana epidermal cell expressing MP:GFP (green channel, left) and BgIG:mRFP (red channel, center). The merged image is shown on the right. d, central view of a $N$. benthamiana epidermal cell expressing MP:GFP-SL (green channel, left) and BgIG:mRFP (red channel, center). The merged image is shown on the right. Arrowheads in $\mathbf{c}$ and $\mathbf{d}$ point to MP:GFP spots at the cell periphery consistent with localization to PD. Arrows in d point to colocalized signals of MP:GFP and BgIG:mRFP indicating the colocalization of MP:GFP and MP:GFPSL mRNA at PD. Scale bars, $5 \mu \mathrm{m}$. e, BgIG:mRFP coimmunoprecipitates with MP:GFP and its SLtagged mRNA. BgIG:mRFP expressed together with either MP:GFP or MP:GFP-SL subjected to coimmunoprecipitation with GFP-specific antibodies. Input (IN), flow-through (FT), and immunoprecipitated (IP) fractions were analyzed with anti-RFP (top) antibodies for detection of BgIG:mRFP and with anti-MP (middle) antibodies for detection of MP:GFP as well as with coomassie blue staining (CBS, bottom), as indicated. Molecular weight is indicated on the left (kDa). 
Arrowheads indicate the positions of BglG:mRFP and MP:GFP. $f$ Quantification of BglG:mRFP immunoreactive signal in the IN, FT, and IP fractions of three independent experiments. The ratio between BgIG:mRFP signal in MP:GFP-SL containing samples and MP:GFP-containing samples is shown. Average values and SD are indicated. ${ }^{*}$, significantly different (unpaired t-test, $p<0,05, n=$ 3).

Figure 4. Cell-to-cell movement of MP mRNA. a, Agroinfiltrated $N$. benthamiana leaf sample expressing BgIG:mRFP in isolated cells and either MP:GFP (left) or MP:GFP-SL (right) in all cells (GFP channel not shown). b, Number of red fluorescent foci consisting of two or more epidermal cells. Average values and SD are shown. ${ }^{*}$, significantly different (unpaired t-test, $p<0,0001, n>400$ ). Data are derived from three experimental replicates. Scale bar, $100 \mu \mathrm{m}$. 


\section{Significance Statement}

Increasing evidence indicates the ability of plants to move RNA species between cells and tissues. Studies to understand the mechanisms by which RNA molecules are transported depend on suitable methods by which the fate of RNA molecules can be addressed in vivo. By adapting components of the bgl operon in Escherichia coli, we achieved unprecedented resolution in localizing tobacco mosaic virus Movement Protein mRNA and tracking its cell-tocell movement in vivo. 

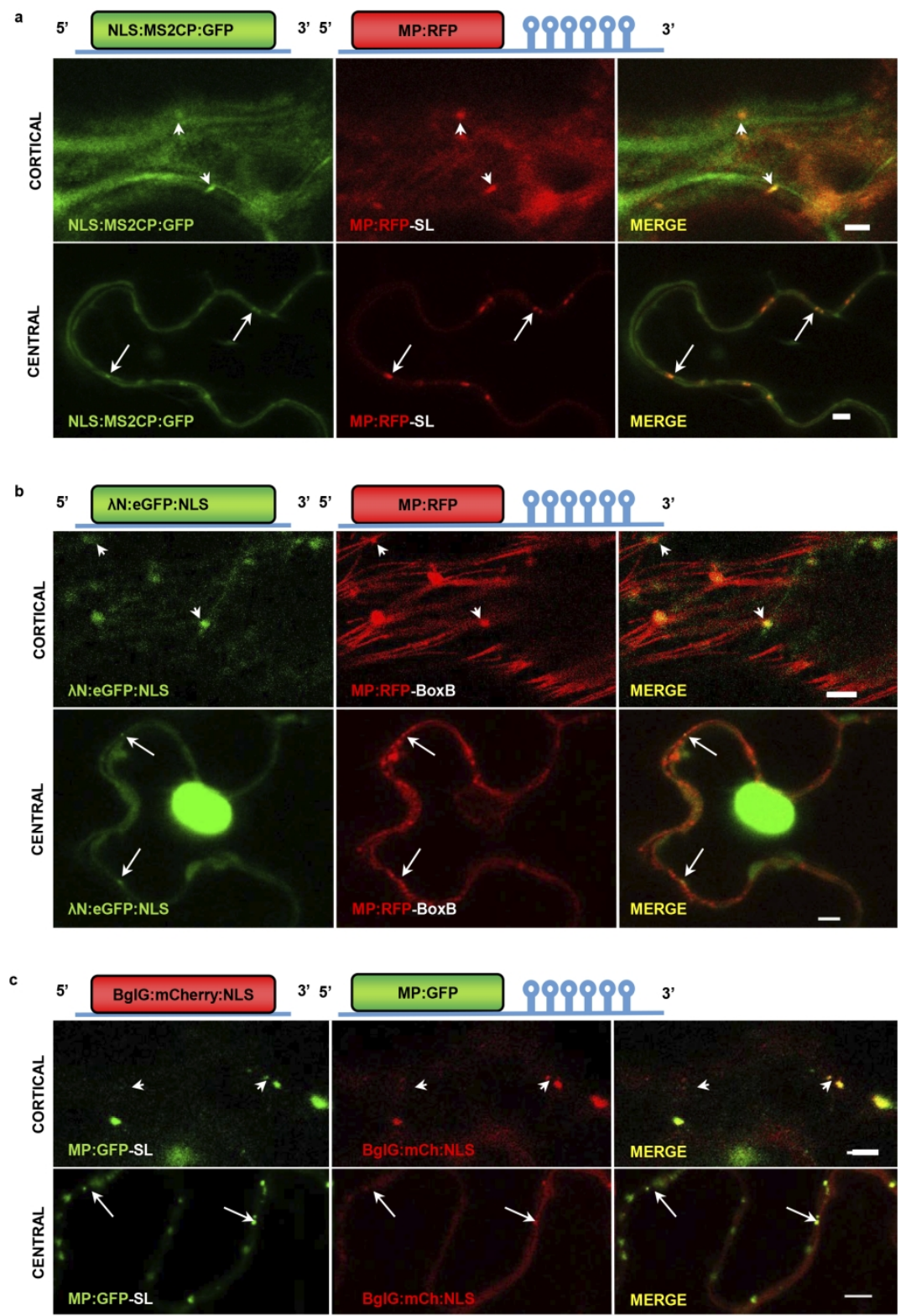

Figure 1. Systems used for the in vivo imaging of TMV MP mRNA. a, MS2 system. Top, representation of transcripts (blue line) and encoded proteins (boxes). Middle, cortical view of a N. benthamiana leaf epidermal cell showing NLS:MS2CP:GFP-labeled MP:RFP mRNA (green channel, left), MP:RFP (red channel, center) and the colocalization of the RNA and protein (yellowish signal in the merged figure, right). The colocalization in MP particles is indicated by arrowheads. Bottom, central view of a cell from the same experiment showing NLS:MS2CP:GFP-labeled MP:RFP mRNA (left) and MP:RFP (center) colocalizing at PD (right; examples of PD localization are indicated by arrows). $b, \lambda N$ system. Top, representation of transcripts (blue line) and encoded protein (boxes). Middle, cortical view of a N. benthamiana epidermal cell showing $\lambda N$ :eGFP:NLS-labeled MP:RFP mRNA (green channel, left), MP:RFP (red channel, center), and colocalization of the RNA and protein (yellow signal in the merged channel, right). Examples of colocalization in MP particles are indicated by arrowheads. Bottom, central view of a cell from the same experiment showing the localization of $\lambda \mathrm{N}$ :eGFP:NLS-labeled MP:RFP mRNA (green channel, left), MP:RFP (red channel, center), and colocalization in the merged image (yellowish signal, right; examples of PD localization are indicated by arrows). c, BglG system. Top, representation of transcripts (blue line) and encoded proteins (boxes). Middle, 
cortical section of a N. benthamiana epidermal cell showing MP:GFP-tagged mobile particles (green, left), BglG:mCherry:NLS-labeled MP:GFP mRNA (red, center) and colocalization of MP:GFP and its mRNA in the merged image (yellow signal, right). Examples of colocalization in MP particles are indicated by arrowheads. Bottom, central view of a cell from the same experiment showing MP:GFP (green, left), BgIG:mCherry:NLSlabeled MP:GFP mRNA (red, center), and the colocalization of MP:GFP mRNA and protein in the merged image (yellow signal, right). Examples of PD localization are indicated by arrows. NLS, nuclear localization signal; SL, stem loops; Scale bars, $5 \mu \mathrm{m}$. 


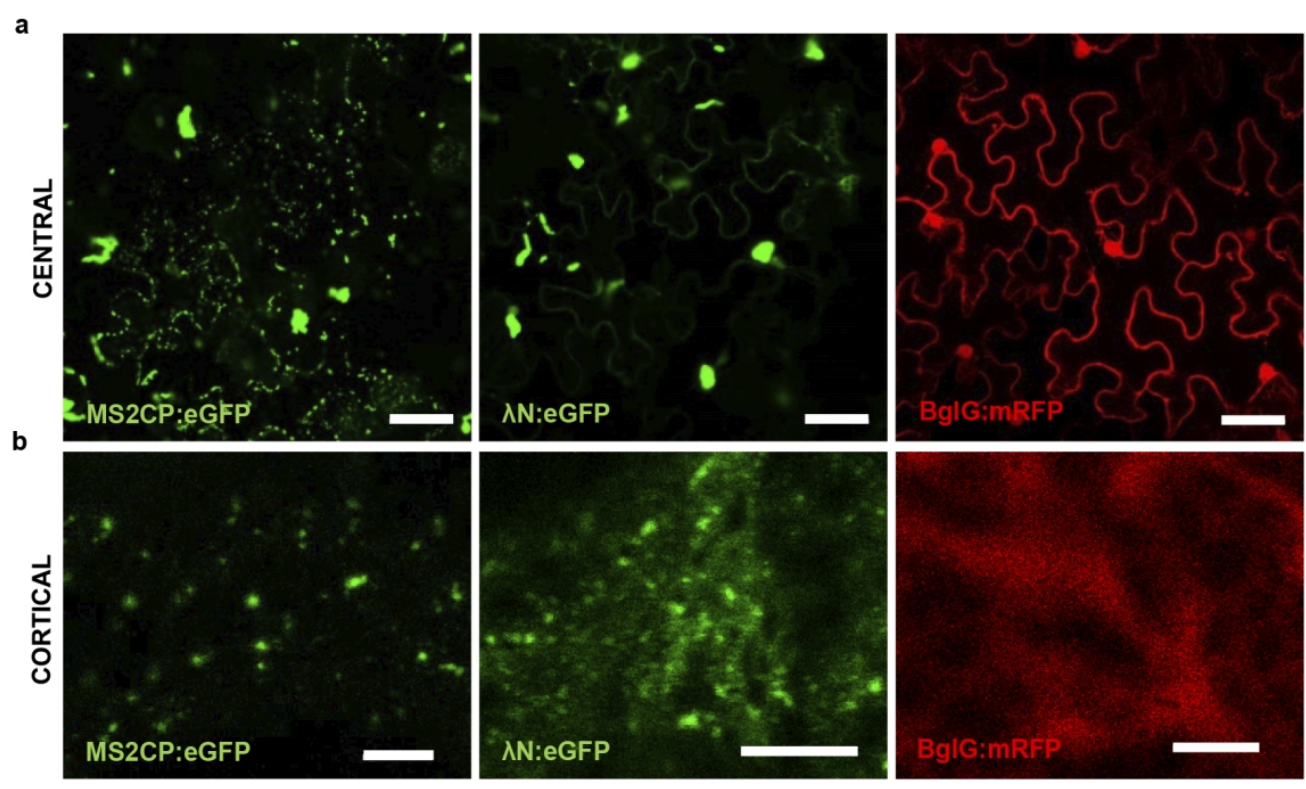

Figure 2. Accumulation patterns of the different RBPs expressed without a NLS. a, Central views of N. benthamiana epidermal cells expressing MS2CP:eGFP (left), $\lambda N$ :eGFP (middle), or BglG:mRFP (right). Scale bar, $50 \mu \mathrm{m}$. b, Cortical views of $\mathrm{N}$. benthamiana epidermal cells expressing MS2CP:eGFP (left), $\lambda \mathrm{N}$ : eGFP (middle), or BglG:mRFP (right). Scale bars, $5 \mu \mathrm{m}$. 
a

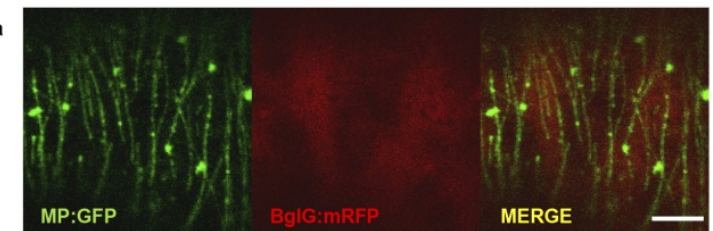

b

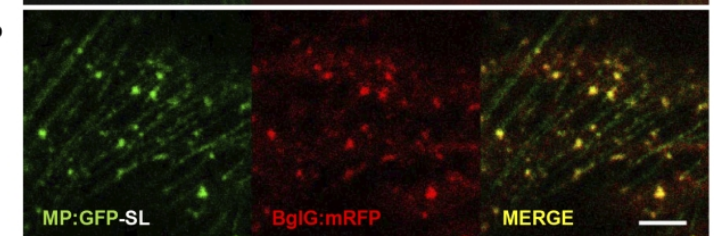

c

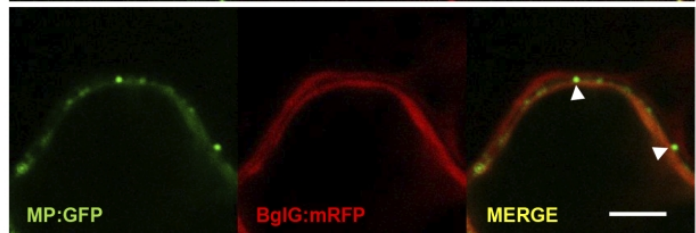

d

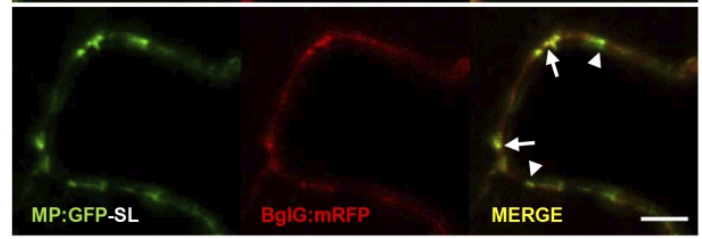

e

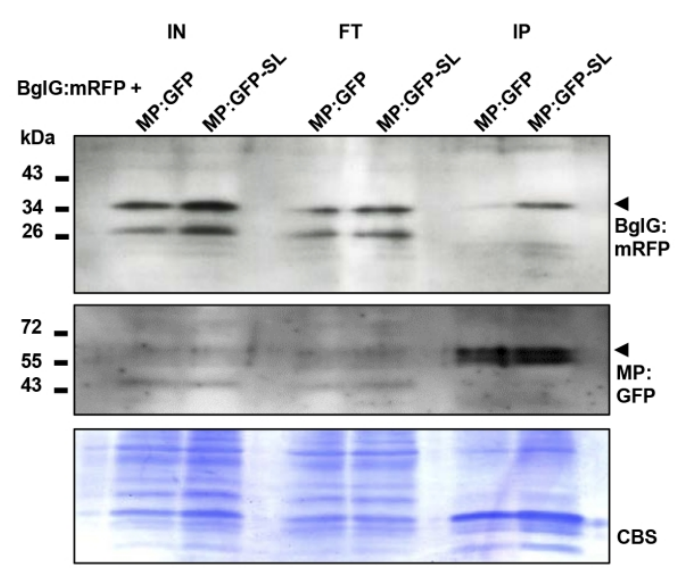

f

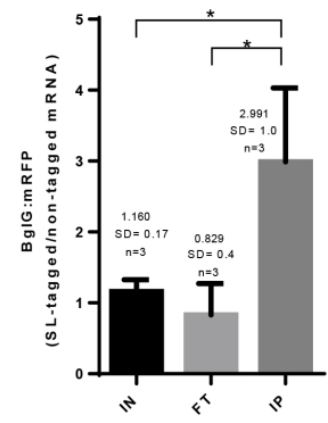

Figure 3. Detection of MP:GFP mRNA using the BglG system. a, cortical view of a N. benthamiana epidermal cell expressing MP:GFP (green channel, left) and BglG:mRFP (red channel, center). The merged image is

shown on the right. $b$, cortical view of a N. benthamiana epidermal cell expressing MP:GFP from mRNA tagged with BglG-SLs (MP:GFP-SL, green channel, left) together with BglG:mRFP (red channel, middle). The merged image shows co-localization of MP:GFP MRNA and protein (yellow signal, right). $c$, central view of a

N. benthamiana epidermal cell expressing MP:GFP (green channel, left) and BglG:mRFP (red channel, center). The merged image is shown on the right. $d$, central view of a $\mathrm{N}$. benthamiana epidermal cell expressing MP:GFP-SL (green channel, left) and BglG:mRFP (red channel, center). The merged image is shown on the right. Arrowheads in $c$ and $d$ point to MP:GFP spots at the cell periphery consistent with localization to PD. Arrows in d point to colocalized signals of MP:GFP and BglG:mRFP indicating the colocalization of MP:GFP and MP:GFP-SL mRNA at PD. Scale bars, $5 \mu \mathrm{m}$. e, BglG:mRFP coimmunoprecipitates with MP:GFP and its SL-tagged mRNA. BglG:mRFP expressed together with either MP:GFP or MP:GFP-SL subjected to coimmunoprecipitation with GFP-specific antibodies. Input (IN), flowthrough (FT), and immunoprecipitated (IP) fractions were analyzed with anti-RFP (top) antibodies for 
detection of BglG:mRFP and with anti-MP (middle) antibodies for detection of MP:GFP as well as with coomassie blue staining (CBS, bottom), as indicated. Molecular weight is indicated on the left (kDa). Arrowheads indicate the positions of BglG:mRFP and MP:GFP. f Quantification of BglG:mRFP immunoreactive signal in the IN, FT, and IP fractions of three independent experiments. The ratio between BglG:mRFP signal in MP:GFP-SL containing samples and MP:GFP-containing samples is shown. Average values and SD are indicated. $*$, significantly different (unpaired t-test, $\mathrm{p}<0,05, \mathrm{n}=3$ ). 
a

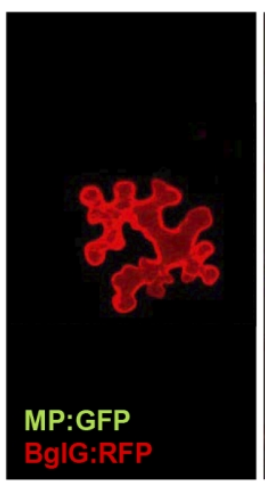

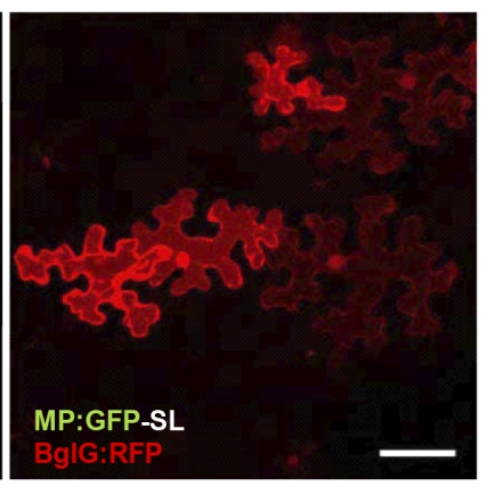

b

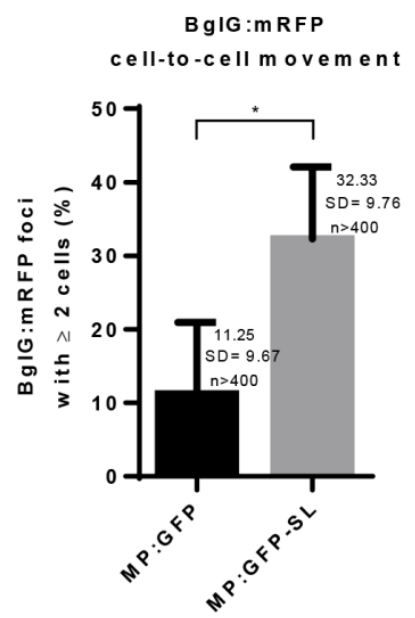

Figure 4. Cell-to-cell movement of MP mRNA. a, Agroinfiltrated N. benthamiana leaf sample expressing BglG:mRFP in isolated cells and either MP:GFP (left) or MP:GFP-SL (right) in all cells (GFP channel not shown). $b$, Number of red fluorescent foci consisting of two or more epidermal cells. Average values and SD are shown. *, significantly different (unpaired t-test, $\mathrm{p}<0,0001, \mathrm{n}>400$ ). Data are derived from three experimental replicates. Scale bar, $100 \mu \mathrm{m}$. 